\title{
El rol de las Clínicas jurídicas en la implementación de los Objetivos de Desarrollo Sostenible (ODS): experiencias desde la Universidad de Oviedo.**
}

\section{Legal Clinic's role in implementing the Sustainable Development Goals (SDGs): experiences from the University of Oviedo}

\author{
Regina H. Fonseca Fortes-Furtado \\ Profa. Asociada de Derecho Penal \\ Departamento de Ciencias Jurídicas Básicas \\ Universidad de Oviedo, España \\ E-mail: fortesregina@uniovi.es
}

\begin{abstract}
Resumen: El movimiento de la Educación Jurídica Clínica se expandió desde Estados Unidos al mundo, así como a más de ciento y cincuenta universidades europeas; en España, a 26 clínicas jurídicas de diversas universidades que integran la Red Española de Clínicas Jurídicas. En este artículo analizaremos cómo potenciar la transferencia de los
\end{abstract}

\footnotetext{
* Este trabajo se desarrolló durante una estancia de investigación en la Universidad de Oporto en los meses de mayo a julio de 2021; agradezco a la Universidad de Oviedo la concesión de una ayuda de movilidad Erasmus+ y a la Universidad de Oporto, en especial al profesor André Lamas Leite y al personal de los Servicios de Movilidad y Biblioteca, su generosa acogida. Quiero agradecer también al profesor Ángel Espiniella, Catedrático de Derecho Internacional Privado de la Universidad de Oviedo, la sugerencia de realizar este estudio.
} 
objetivos de desarrollo sostenible de Naciones Unidas (ODS) a la sociedad civil, profesional, académica, etc., a través del uso de la metodología clínica de educación jurídica, con especial atención al Proyecto de Innovación Docente coordinado de las clínicas de la Universidad de Oviedo, que pretende la introducción de los ODS en el aprendizaje-servicio de las clínicas jurídicas.

Palabras clave: Educación jurídica clínica, Clínicas Jurídicas, Objetivos de Desarrollo Sostenible, Educación en valores, Proyecto de innovación docente.

Abstract: The Clinical Legal Education movement expanded from the United States to the world, as well as to more than one hundred and fifty European universities, among which Spain currently has the Spanish Network of Legal Clinics that adds twenty-six legal clinics from various Spanish universities. In this article we will analyse how to promote the transfer of the Sustainable Development Goals of the United Nations (SDGs) to civil society, professional, academic, etc., through the use of the clinical methodology of legal education, with special attention to the Coordinated Teaching Innovation Programme (TIP) of the Legal Clinics of the University of Oviedo that deals with the introduction of the SDGs in the learning-service of legal clinics.

Keywords: Clinical legal education, Sustainable Development Goals, Values in education, Teaching innovation program.

Sumario: 1. La Educación Clínica Jurídica. 2. Las Clínicas Jurídicas en España y la implementación de los Objetivos de Desarrollo Sostenible. 3. El Proyecto de Innovación Docente coordinado de las Clínicas Jurídicas de la Universidad de Oviedo. 3.1. El "ex ante": líneas básicas del Proyecto de Innovación Docente coordinado de las Clínicas Jurídicas de la Universidad de Oviedo. 3.2. El "ex post": el desarrollo de actividades en el ámbito del Proyecto de Innovación Docente coordinado. 3.3. Balance provisional. 4. Bibliografía.

\section{La Educación Clínica Jurídica}

El movimiento de educación jurídica clínica (Clinical Legal Education o CLE) despega en los años 30 del siglo pasado en Estados Unidos bajo el influjo del realismo jurídico 
norteamericano y de una de sus principales figuras, el magistrado y profesor Jerome Frank, contraponiéndose al formalismo jurídico del library-law del profesor Christopher C. Langdell -duramente criticado por el primero-, que centraba el estudio del Derecho en la resolución de casos a través de la utilización de libros, método también conocido como case system o Harvard System, por haber sido adoptado por esta prestigiosa universidad (WITKER, 2007).

Langdell defendía que todo lo que se necesita para enseñar Derecho "se encuentra impreso en libros", renegando de cualquier otra fuente que no estuviese en las bibliotecas de las universidades. Por su parte, Frank señalaba que el estudio del Derecho a través de casos presentaba tres problemas insolubles: en primer lugar, el Derecho se enseña como algo estático, alejado de las transformaciones sociales; en segundo término, se estudia el caso, que es el fin de un proceso, sin contextualizar el Derecho como medio para alcanzar determinados fines, con todos los problemas que experimenta durante este recorrido; finalmente, censura la ausencia o poca relación que hay entre los casos estudiados ("casos de laboratorio") y la realidad de las calles (FRANK, 1933).

La enseñanza del Derecho en las universidades norteamericanas adolecía de un academicismo elitista, sesgado y alejado de la realidad social, en el que los estudiantes no tenían que pensar, sino que debían limitarse a replicar la información que ya se encontraba plasmada en los libros de Derecho, mientras que los buenos docentes deberían encontrarse en las bibliotecas y no en los despachos de abogados. En fin, se pensaba que la práctica contaminaba a la academia y le quitaba el carácter científico que solo se podría obtener con docentes que, a su vez, no estuviesen contaminados por la práctica en los bufetes y los juzgados y tribunales. Por otro lado, los estudiantes salían de las universidades y se encontraban con un abismo entre la teoría y la práctica, que los conducía a una caída inevitable o a la paralización para evitarla.

En un giro copernicano, Frank propuso un acercamiento al modelo de las clínicas gratuitas y de los dispensarios utilizados por las Facultades de Medicina, con una enseñanza práctica del Derecho mediante "Clínicas Jurídicas” ubicadas en las Facultades de Derecho, en las que los estudiantes, supervisados por docentes o profesionales del Derecho, mantuviesen un contacto con casos reales y prestasen asistencia jurídica, de forma gratuita, a personas sin recursos económico. 
El contexto histórico por el que pasaba Estados Unidos, el de las consecuencias de la gran crisis de 1929, ciertamente contribuyó a que las Universidades encontrasen en las clínicas jurídicas un espacio para cumplir con unos cometidos de responsabilidad social y transformación e interacción con la sociedad; también la financiación concedida por la Fundación Ford al programa Council on Legal Education for Professional Responsability (CLEPR) fue decisiva para que varias universidades adoptasen la metodología de educación clínica. En los años 60 y 70 del pasado siglo el movimiento clínico se extendió rápidamente a otros países de habla inglesa, como Gran Bretaña, Canadá y Australia, coincidiendo con los movimientos de lucha por los derechos civiles, el pacifismo, el feminismo, la incipiente cooperación al desarrollo y la promoción de la ética profesional (BLOCH, 2010, 2012).

En la Europa continental la metodología clínica de educación jurídica empezó de la mano de los cambios sociopolíticos derivados de la caída del Muro de Berlín y de los esfuerzos para consolidar la democracia y el Estado de Derecho en los países de Europa del Este. Con la posterior creación de un sistema común europeo de enseñanza y del Espacio Europeo de Enseñanza (EEE), así como con la consiguiente implantación del llamado "Plan Bolonia", las universidades europeas atendieron a la creciente demanda de una educación jurídica profesional que incluyera una aplicación práctica de los conocimientos, centrada en la capacidad de análisis y solución de problemas jurídicos y conectada con la realidad social.

En la actualidad, la red global European Network for Clinical Legal Education (ENCLE) cuenta con más de 150 integrantes de 20 países y varias universidades afiliadas alrededor del mundo. En el mes de junio del año 2021 se celebró de forma telemática la Conferencia Mundial de las clínicas jurídicas con el tema "Turning Challenges into Opportunities: Justice Education in Times of Crisis", organizada por la Northumbria University, de Inglaterra ${ }^{114}$.

La globalización y la conexión a través de redes utilizando la tecnología de la información facilitaron la llegada de la CLE a África, sudeste de Asia e India, América Latina, China

${ }^{114}$ https://gaje.org/Upcoming-Conferences 
y Japón, y el modelo de las clínicas jurídicas es, hoy por hoy, mundialmente reconocido y utilizado en varias instituciones de enseñanza universitaria.

\section{Las Clínicas Jurídicas en España y la implementación de los Objetivos de Desarrollo Sostenible}

España cuenta con su propia red de clínicas jurídicas, que se constituyó a partir de los encuentros realizados en las Universidades Rovira i Virgili, de Tarragona (2007; en la Universidad Carlos III, de Madrid (2014); en la Universitat de València (2010, 2013 y 2016) y en la Universidad de Alcalá (2017), y que cuenta actualmente con 26 clínicas jurídicas asociadas (RED ESPAÑOLA DE CLÍNICAS JURÍDICAS, 2021).

No hay un modelo único de clínicas jurídicas, pues estas entidades se adaptan a los objetivos, las necesidades y al entorno cultural y geográfico, por lo que pueden adoptar diferentes formas: asesoría jurídica, representación jurídica, clínicas de divulgación jurídica “en la calle” -Street Law Clinics- (PRESNO LINERA, 2018), resolución alternativa de conflictos, servicios jurídicos comunitarios, abogacía de interés público, etc. ${ }^{115}$

Las universidades tienen total libertad para adoptar el modelo que más encaje en su perfil; no es nuestro objetivo profundizar en cada modelo, sino analizar brevemente las actividades de las clínicas jurídicas españolas con relación a un ámbito específico de actuación: la utilización de los Objetivos de Desarrollo Sostenible de Naciones Unidas (ODS) en sus programas clínicos.

Como es conocido, mediante un nuevo contrato social global que incluye a todos los países -los ricos y los pobres- los Objetivos de Desarrollo Sostenible se crearon con la finalidad de fomentar la igualdad entre las personas, proteger el planeta y asegurar la prosperidad como parte de la Agenda 2030 de compromiso con el desarrollo sostenible a nivel planetario, a diferencia de sus precursores, los Objetivos de Desarrollo del Milenio $(\mathrm{ODM})$, que se centraran principalmente en los países en desarrollo, en particular los más

\footnotetext{
${ }^{115}$ Cfr. también los monográficos dedicados al tema de las Clínicas Jurídicas: AA.VV. (2011) "Clínicas Jurídicas: Desafío y oportunidad", Revista Educación y Derecho (4) y AA.VV. (2015) "Modelos de Clínicas Jurídicas en España”, Revista Educación y Derecho (11).
} 
pobres, con una agenda mayoritariamente social, proponiendo la reducción de la pobreza extrema y de las tasas de mortalidad infantil, la lucha contra epidemias de enfermedades como el VIH/SIDA y una alianza mundial para el desarrollo.

La Resolución 66/288, del año 2012, adoptada por la Asamblea General de Naciones Unidas $^{116}$, fue el punto de partida para la presentación de una Agenda Internacional de Desarrollo y de los ODS en la Conferencia sobre Desarrollo Sostenible Rio+20, en la que se creó un grupo de trabajo a partir del cual se elaboraron encuestas a la ciudadanía de diversos países preguntando qué consideraban que podría cambiar su vida, y los objetivos más votados fueran una buena educación, mejor atención médica, gobierno honesto y mejores oportunidades de trabajo. Tras un año de negociaciones el grupo de trabajo recomendó la adopción de 17 objetivos y de 169 metas para el período comprendido entre 2015-2030, de ahí la Agenda 2030 y los 17 ODS: fin de la pobreza, hambre cero, salud y bienestar, educación de calidad, igualdad de género, agua limpia y saneamiento, energía asequible y no contaminante, trabajo decente y crecimiento económico; industria, innovación e infraestructura; reducción de las desigualdades, ciudades y comunidades sostenibles, producción y consumo responsables, acción por el clima, vida submarina, vida de ecosistemas terrestres; paz, justicia e instituciones sólidas, alianzas para lograr los objetivos.

El impacto de los ODS en las clínicas jurídicas españolas se hizo sentir en un espectro de distinta intensidad y diferentes enfoques transversales, variando en virtud de los temas a que se dedican y de los tipos de clínicas jurídicas de que se trate.

La metodología que hemos aplicado a nuestro estudio se ha basado en, preguntar, a través de la propia Red Española de Clínicas Jurídicas, sobre la aplicación de los ODS por las mencionadas clínicas jurídicas; a continuación, se pueden ver de forma gráfica los resultados obtenidos:

\footnotetext{
${ }^{116}$ A/RES/66/288, 27 de julio 2012, The future we want. Disponible en: A/RES/66/288: The future we want (un.org), acceso el 08/06/2021.
} 
Tabla 1 - Utilización de los ODS en las Clínicas Jurídicas Españolas

\begin{tabular}{c|ccc} 
& \multicolumn{1}{c}{$\begin{array}{c}\text { ÁMBITO DE } \\
\text { APLICACIÓN }\end{array}$} & LOCALIDAD & PROYECTOS Y ODS \\
\hline $\begin{array}{c}\text { Universidad } \\
\text { Carlos III }\end{array}$ & $\begin{array}{c}\text { Clínica Jurídica del } \\
\text { Instituto de Derechos } \\
\text { Humanos "Bartolomé } \\
\text { de las Casas" }\end{array}$ & Madrid & ODS y discapacidad \\
\hline
\end{tabular}

\begin{tabular}{c|c} 
Universidad & $\begin{array}{c}\text { Clínica Jurídica de } \\
\text { derechos humanos } \\
\text { Carlos III }\end{array}$ \\
Javier Romañach
\end{tabular}

Universidad

Javier Romañach

Proyecto "Los ODS, Agenda 2030 en clave de Convención Internacional sobre los derechos de las personas con discapacidad: una relectura para el refuerzo mutuo de ambas agendas"

(En colaboración con CERMI Estatal) ODS $10^{*}$

Enfoque Basado en Derechos

\begin{tabular}{c|c|c}
$\begin{array}{c}\text { Universidad de } \\
\text { Valladolid }\end{array}$ & $\begin{array}{c}\text { Clínica Jurídica y en el } \\
\text { Observatorio de } \\
\text { derechos humanos }\end{array}$ & Valladolid \\
\hline
\end{tabular}

Humanos para poner en marcha políticas públicas, entre ellas las de cooperación ODS $16^{*}$

Aprendizaje Servicio del Máster en

Derecho de la empresa y de la contratación. El curso pasado los alumnos realizaron un dictamen para un ateneo de cooperativas con el

\begin{tabular}{c|c|c} 
Universitat & $\begin{array}{c}\text { Clínica Jurídica de la } \\
\text { Universitat Rovira i } \\
\text { Rovira i Virgili }\end{array}$ & Tarragona \\
\hline
\end{tabular}
objetivo de adaptar las cooperativas a los ODS, fundamentar la conveniencia y detallaron un plan de actuación para una de sus cooperativas concretas a modo de ejemplo ODS $9 *$

\begin{tabular}{|c|c|c|c|}
\hline $\begin{array}{l}\text { Universitat Illes } \\
\text { Balears }\end{array}$ & Clínica Jurídica UIB & $\begin{array}{l}\text { Palma de } \\
\text { Mallorca }\end{array}$ & $\begin{array}{c}\text { Proyecto de innovación docente sobre } \\
\text { aprendizaje de Derechos humanos } \\
\text { Proyecto de investigación sobre } \\
\text { acceso de solicitantes de asilo, en } \\
\text { Baleares, a petición de la oficina de } \\
\text { cooperación de la UIB } \\
\text { ODS } 16\end{array}$ \\
\hline $\begin{array}{l}\text { Universidad de } \\
\text { Salamanca }\end{array}$ & $\begin{array}{l}\text { Clínica Jurídica de } \\
\text { Acción Social }\end{array}$ & Salamanca & $\begin{array}{l}\text { Medio ambiente y consumo } \\
\text { responsable } \\
\text { ODS } 12 *\end{array}$ \\
\hline $\begin{array}{l}\text { Universidad del } \\
\text { País Vasco }\end{array}$ & $\begin{array}{c}\text { Clínica Jurídica para la } \\
\text { Justicia Social }\end{array}$ & Leioa & $\begin{array}{c}\text { Proyecto de Innovación Educativa } \\
\text { para el bienio 2021-2022 ODS 5, } 10 \text { y } \\
16\end{array}$ \\
\hline $\begin{array}{l}\text { Universidad } \\
\text { Europea de } \\
\text { Madrid }\end{array}$ & $\begin{array}{c}\text { Clínica Jurídica de la } \\
\text { Universidad Europea de } \\
\text { Madrid }\end{array}$ & Madrid & $\begin{array}{c}\text { Informe } \\
\text { (En colaboración con la Fundación } \\
\text { Fernando Pombo) } \\
\text { ODS 5, } 8 \text { y } 16\end{array}$ \\
\hline $\begin{array}{c}\text { Universitat } \\
\text { Jaume I }\end{array}$ & $\begin{array}{l}\text { Clínica Jurídica de la } \\
\text { Universitat Jaume I }\end{array}$ & $\begin{array}{c}\text { Castellón de la } \\
\text { Plana }\end{array}$ & $\begin{array}{l}\text { Espacio Jurídico de Estudios } \\
\text { Laborales (Clínica jurídica y } \\
\text { laboratorio docente) Actividad y } \\
\text { proyectos } \\
\text { ODS } 5,8 \text { y } 10\end{array}$ \\
\hline $\begin{array}{l}\text { Universidad de } \\
\text { Oviedo }\end{array}$ & $\begin{array}{l}\text { Clínica Jurídica Uniovi } \\
\text { y Clínica Jurídica del } \\
\text { Máster en Protección de } \\
\text { Grupos Vulnerables }\end{array}$ & Oviedo & $\begin{array}{l}\text { Proyecto de Innovación Docente de } \\
\text { las dos clínicas } \\
\text { ODS (todos) }\end{array}$ \\
\hline
\end{tabular}

Fuente: elaboración propia.

* ODS no especificado en las consultas respondidas. 
Como se puede ver en esta tabla, 11 Clínicas aplican los ODS (42,3\%), mientras que 15 Clínicas no utilizan los ODS o, al menos, no informaron sobre su utilización (57,69\%), lo que nos permite concluir que hay grandes posibilidades de ampliación de la utilización de los ODS en el ámbito de las Clínicas jurídicas españolas.

Por otro lado, con relación a los ODS comunicados por las Clínicas, hay que poner de relieve que algunas de ellas especificaron cuáles eran, mientras que otras no lo hicieron. A las que no lo hicieron, hemos tenido que asignar un ODS para cada tipo de proyecto; por ejemplo, al tema de la discapacidad le hemos asignado el ODS 10 (reducción de las desigualdades), al de las cooperativas el ODS 9 (industria, innovación e infraestructura) y los relacionados con Derechos humanos el ODS 16 (paz, justicia e instituciones sólidas), a medio ambiente y consumo responsable el ODS 12 (producción y consumo responsables); con la siguiente distribución gráfica de los ODS en el ámbito de las Clínicas Jurídicas que los utilizan resulta lo siguiente:

\section{Gráfico 1}

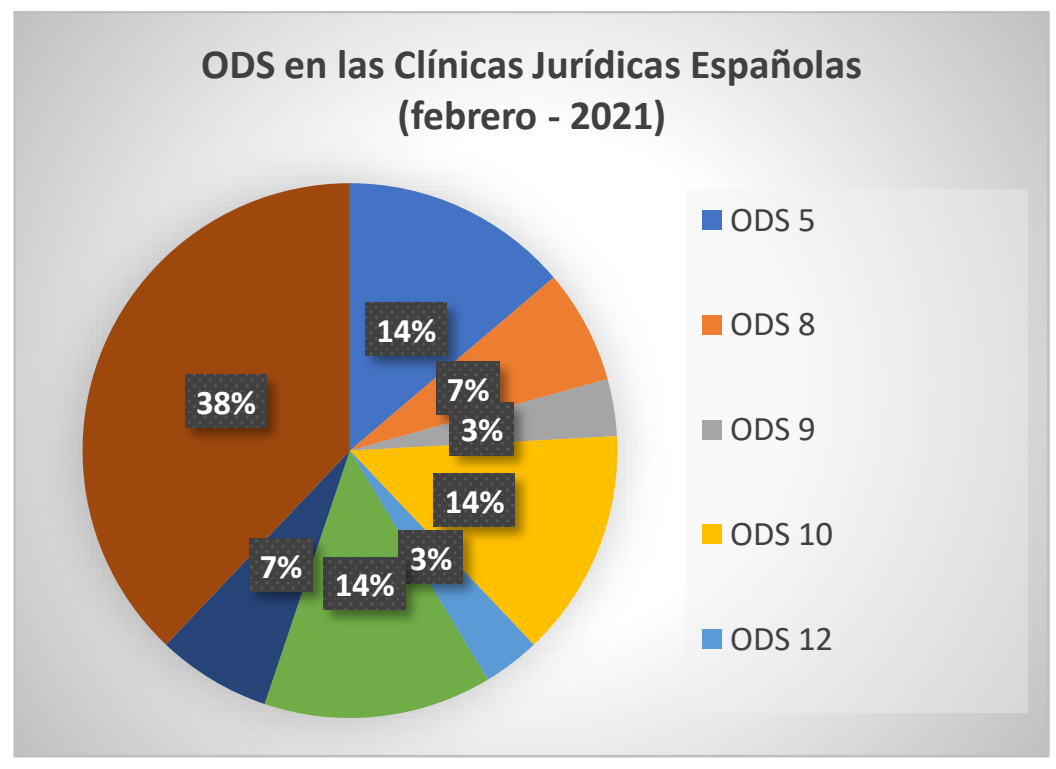

Fuente: elaboración propia a partir de los datos de la tabla anterior

Inicialmente, hemos constatado que, de acuerdo con la distribución de los ODS en el ámbito de los proyectos desarrollados por las Clínicas Jurídicas españolas, a) hay muy poca transversalidad en su uso (los ODS pueden y deben combinarse entre sí); b) también es poca la utilización del ODS 4 Educación de calidad (que podría ser utilizado en 
prácticamente todas las actividades de las Clínicas Jurídicas); c) la no utilización del ODS 17 Alianza para lograr los objetivos; de hecho solamente en dos de los proyectos se menciona la colaboración con alguna institución, que podría tratarse, o no, de una alianza a los efectos aquí tratados; d) hay varios ODS (38\%) que no se mencionan expresamente, pero que están en el Proyecto de Innovación Docente de las Clínicas Jurídicas de la Universidad de Oviedo, que utilizan todos los ODS (un 7\%) del total; e) la porción de la "tarta" (38\%) de los ODS que no se asignan, excepto en los dos proyectos de la Universidad de Oviedo que utilizan todos los ODS, la forman los ODS 1 (fin de la pobreza), 2 (hambre cero), 3 (salud y bienestar), 4 (educación de calidad), 6 (agua limpia y saneamiento), 7 (energía asequible y no contaminante), 11 (ciudades y comunidades sostenibles), 13 (acción por el clima), 14 (vida submarina), 15 (vida de ecosistemas terrestres), y 17 (alianzas para lograr los objetivos).

No obstante, esta última afirmación hay que matizarla, pues el hecho de que no se asigne un ODS a un proyecto no significa que no esté de facto incluido en él. Como hemos mencionado, prácticamente todos los proyectos pueden incluirse en el ODS 4 educación de calidad, mientras que también muchos de ellos implican alianzas para la consecución de los objetivos propuestos.

A continuación, nos parece interesante exponer con más detalle el Proyecto de Innovación Docente coordinado llevado a cabo durante el curso académico 2020-2021 por las Clínicas Jurídicas de la Universidad de Oviedo y ello por los siguientes motivos: a) en la actualidad es el que más ampliamente utiliza a los ODS; b) ayuda a fomentar la transferencia de los ODS a la sociedad civil, profesional y académica; c) puede servir de inspiración para otros proyectos, por su fácil adaptabilidad a diferentes formatos y tipos de clínicas jurídicas; d) es un proyecto en el que hemos trabajado, lo que nos permite hacer una crítica a sus posibles lagunas para poder perfeccionar eventuales futuros proyectos.

3. El Proyecto de Innovación Docente coordinado de las Clínicas Jurídicas de la Universidad de Oviedo

Señala GARCÍA AÑÓN $(2015,1)$ tres aspectos importantes de la educación jurídica clínica como instrumento de transformación de la enseñanza del Derecho: el cambio de 
los métodos de enseñanza, el papel de la Universidad en y para la sociedad y la colaboración con el acceso a la Justicia y a los derechos, desde -pero no solo- la perspectiva de la formación. Los distintos modelos de clínicas jurídicas que encontramos en España se basan en estos aspectos de innovación docente y de responsabilidad social de la Universidad señalados por el autor, de entre los que encontramos los Proyectos de Innovación Docente coordinados de las dos clínicas jurídicas de la Universidad de Oviedo que analizaremos a continuación. ${ }^{117}$

\subsection{El "ex ante”: líneas básicas del Proyecto de Innovación Docente coordinado de las Clínicas Jurídicas de la Universidad de Oviedo}

El Proyecto coordinado de innovación docente en las Clínicas Jurídicas de la Universidad de Oviedo introduce los objetivos de desarrollo sostenible en la enseñanza del Derecho a través de la metodología de aprendizaje-servicio de las Clínicas jurídicas, en las que los estudiantes realizan servicios de asesoría, elaboración de informes y dictámenes, divulgación social, confección de guías de actuación y actividades solidarias. Se incluyen 17 acciones específicas y 9 acciones transversales, siendo una de sus principales fortalezas la implicación de la totalidad de áreas de conocimiento jurídico de la Universidad de Oviedo y de sus tres departamentos jurídicos. Además de la señalada innovación docente, se busca cierta innovación social, por la identificación de una necesidad de actuación en la sociedad asturiana sobre desarrollo sostenible. Todo ello se completa con una innovación institucional, pues por primera vez se presenta un proyecto coordinado con dos subproyectos vinculados a distintas titulaciones y en distintas etapas de grado y postgrado.

Los objetivos generales son: impulsar decididamente el aprendizaje activo del estudiantado, dar la máxima visibilidad y proyección social a las actividades clínicas, convertir a la Clínicas Jurídicas en un referente para la sociedad asturiana enla Agenda 2030 y coordinar las actuaciones de las clínicas de Grado y Máster.

117 "La introducción de los Objetivos de Desarrollo Sostenible en el aprendizaje-servicio de la Clínica Jurídica de Grado UNIOVI" (PINN-20-037) y "La introducción de los Objetivos de Desarrollo Sostenible en el aprendizaje-servicio de la Clínica Jurídica del Máster en protección jurídica de las personas y los grupos vulnerables" (PINN-20-A-054). 
En lo que respecta a los objetivos específicos, el proyecto pretende introducir los objetivos de desarrollo sostenible en el aprendizaje del Derecho, mejorar los resultados de ese aprendizaje a través de la metodología clínica de “aprendizaje por servicio", formar profesionales del ámbito jurídico con competencias específicas en materia de desarrollo sostenible, diferenciar objetivos de desarrollo sostenible en función de su construcción en clave de vulnerabilidad y crear en las clínicas un espacio solidario en aspectos relacionados con elobjetivo de desarrollo sostenible.

Los dos subproyectos cuentan con coordinadores, subcoordinadores y directores y en ambos están representadas las áreas del Departamento de Ciencias Jurídicas Básicas (Derecho Romano, Derecho Eclesiástico del Estado, Derecho Procesal, Derecho Penal, Filosofía del Derecho e Historia del Derecho), del Departamento de Derecho Privado y de la Empresa (Derecho Civil,Derecho del Trabajo y de la Seguridad Social, Derecho Mercantil y Derecho Internacional Privado), y del Departamento de Derecho Público (Derecho Constitucional, Derecho Internacional Público y Relaciones Internacionales, Derecho Administrativo y Derecho Financiero y Tributario); asimismo, la Biblioteca de Ciencias Jurídico-Sociales presta apoyo con la cesión de espacios, el impulso de actividades solidarias o relacionadas con el ODS 4 sobre una educación de calidad y la recomendación en redes sociales de novedades bibliográficas relacionadas con la actividad de las clínicas.

La metodología docente adoptada ha sido la siguiente: a) la sensibilización en relación con una necesidad social, ambiental, económica... identificada a partir de los objetivos de desarrollo sostenible y de un concreto incumplimiento o demanda en la sociedad asturiana; b) el compromiso de mejorar la situación o reducir la necesidad a través de un proyecto de acción, un proyecto educativo con finalidad social. Las actuaciones adoptan alguna de las siguientes modalidades:

b.1) Asesorar por parte del estudiantado a operadores sociales o institucionales que lo soliciten.

b.2) Organizar jornadas o debates en los que el estudiantado participeactivamente en su proyección a la sociedad. 
b.3) Elaborar informes sobre desarrollo sostenible de acuerdo con concretos operadores.

b.4) Elaborar guías de actuación y material multimedia para la ciudadanía en materia diversas: atención social, consumo responsable, medio ambiente, etc.

b.5) Realizar talleres para abordar necesidades legislativas y, en la medida de lo posible, influir en la toma de decisiones legislativas.

b.6) Continuar con la metodología de Street Law de la Clínica del Máster y organizar dinámicas en institutos y colegios, que ha funcionado de forma muy destacada desde su inicio en 2015.

b.7) Fomentar iniciativas solidarias por parte del estudiantado sobre el esquema aprendizaje-servicio.

Se sigue un extenso "Plan de Trabajo" con 17 acciones específicas, una para cada ODS y 09 acciones transversales, tales como la presentación de las clínicas jurídicas al inicio de curso, fomentar la participación de los estudiantes en grupos transversales de trabajo, elaboración de memoria y de organigrama, etc., además de la asignación de mentores para cada acción y algunas fechas aproximadas para desarrollo de las actividades.

Los resultados buscados son la implantación de una innovación docente, vinculada a la metodología aprendizaje-servicio del Derecho a través de la clínica jurídica, que permite un servicio a operadores de la sociedad asturiana a través de un proyecto educativo solidario y la implantación de una innovación social, por la identificación de una necesidad de actuación en la sociedad asturiana.

El proyecto también aspira a la innovación institucional a través de la coordinación de dos subproyectos vinculados a distintas titulaciones y en distintas etapas: Grado en Derecho y Máster en Grupos Vulnerables, y con ello pretende la formación de equipos mixtos, que incluyan estudiantes de grado y posgrado, logrando una nueva versión del aprendizaje entre iguales y la presencia de posibles alumnos mentores. 
Además, se buscan también resultados de aprendizaje especialmente significativos en la adquisición de competencias generales (expresión oral y escrita, liderazgo, organización, trabajo en grupo...), de competencias específicas en el ámbito del desarrollo sostenible y las Ciencias jurídicas básicas, el Derecho público y el Derecho privado y de la empresa; una o más publicaciones especializadas en la materia; dos presentaciones en congresos; diecisiete actividades de proyección social, al menos una por objetivo de desarrollo sostenible y la organización de un congreso sobre la metodología clínica de aprendizajeservicio en el Derecho.

Por fin, se incluyen los indicadores de evaluación del proyecto: número de estudiantes y profesores participantes, entidades externas colaboradoras, medición del grado de satisfacción a través de encuestas, calidad e indexación de la publicación o publicaciones resultado del proyecto, relevancia nacional de la presentación del proyecto en la Red Española deClínicas, relevancia internacional de la presentación del proyecto en la Red Europea deClínicas, aceptación de las ponencias por el comité científico del congreso nacional, aceptación de las ponencias por el comité científico internacional.

Finalmente, consta un listado extenso y abierto de las entidades colaboradoras del que llama la atención la cantidad de organizaciones que pueden estar involucradas en las actividades propuestas.

\subsection{El "ex post": el desarrollo de actividades en el ámbito del Proyecto de Innovación} Docente coordinado

Durante el curso 2020-2021 han estado activas doce actividades en el ámbito del Proyecto de Innovación Docente coordinado de las Clínicas de la Universidad de Oviedo: el Proyecto sobre internamientos involuntarios con la Asociación de Familiares y Personas con Enfermedad Mental de Asturias (AFESA), el Proyecto con la Asociación Hierbabuena para analizar si las propuestas de reforma legislativa que se han estado debatiendo en las Cortes Generales son conformes con la Convención sobre derechos de las personas con discapacidad, el Proyecto sobre la trata de personas con Amnistía Internacional, el Proyecto para el estudio de las órdenes de protección por violencia doméstica y/o de género en Asturias con el Ilustre Colegio de Abogados de Oviedo (ICAO), el Proyecto con la Fundación Secretariado Gitano para el acercamiento a 
problemas jurídicos de la comunidad gitana, el Proyecto sobre los derechos de las personas enfermas de cáncer y acompañantes con la Asociación Española contra el Cáncer (AECC), el Proyecto con la University of Miami School of Law Human Rights Clinic, el Proyecto Covidlab con la Universidad de Georgetown y el instituto O’Neill de Derecho de la Salud Nacional y Global, el proyecto Moot Court Competition con la The European Law Students’ Association (ELSA Spain), el Proyecto con la Clínica Jurídica de la Universidad de Alcalá para la elaboración del Informe "Libertad de expresión, delitos de odio y VIH: a propósito de un cartel" y el Proyecto sobre sanciones de la Unión Europea por violación de Derechos Humanos, en colaboración con la subcomisión de Derechos Humanos del Parlamento Europeo. ${ }^{118}$

No podemos extendernos en los doce proyectos que acabamos de mencionar, pero nos gustaría destacar algunos de ellos, como el de colaboración con la Clínica Jurídica de Derechos Humanos de la Miami Law School, que tiene como objetivo realizar un informe sobre la situación de las mujeres indígenas en Guatemala y que abordará el derecho a la alimentación, a la participación pública, a la defensa de los derechos, a la protección frente a la violencia y el acceso a las instituciones defensoras de derechos humanos. El informe se redactará teniendo en cuenta el marco normativo internacional, regional y guatemalteco sobre estas materias, y tras la realización de entrevistas a mujeres indígenas guatemaltecas para conocer la realidad de la aplicación de las normas; en esta actuación participarán seis estudiantes, tanto del Grado en Derecho, como del doble Grado en Derecho y Administración de Empresas.

Otro es el de colaboración con el Instituto O’Neill de Derecho de la Salud Nacional y Global (O’Neill Institute for National \& Global Health Law) de la Universidad de Georgetown, para permitir a los estudiantes participar en el COVID-19 Law Lab, la mayor base de datos de normas y otros documentos de los estados relacionados con la

\footnotetext{
${ }^{118}$ En este apartado me sirvo de varios informes: en primer lugar, del elaborado por FERNANDEZ-RIVERA GONZÁLEZ, M.P. y GARCíA ÁMEZ, J. Clínica Jurídica de la Universidad de Oviedo. Disponible en: Facultad de Derecho - Universidad de Oviedo - Presentación (uniovi.es), acceso el 11/06/2021; cfr. el enlace sobre el funcionamiento y actividades de la CJUO en la página web de presentación de la clínica donde se puede consultar la información sobre los proyectos y actividades mencionados en el ámbito de este estudio; también pude disponer de las Memorias Finales de los dos proyectos de innovación docente coordinados reconocidos por la Universidad de Oviedo a sus clínicas jurídicas: "La introducción de los Objetivos de Desarrollo Sostenible en el aprendizaje-servicio de la Clínica Jurídica de Grado UNIOVI" (PINN-20-037) y "La introducción de los Objetivos de Desarrollo Sostenible en el aprendizaje-servicio de la Clínica Jurídica del Máster en protección jurídica de las personas y los grupos vulnerables” (PINN-20-A-054).
} 
pandemia de la COVID-19. Los estudiantes colaborarán no solo en la labor de búsqueda normativa, sino que, además, adquirirán conocimientos sobre la aplicación del Derecho en épocas de pandemia y las formas de intervención para atajar los distintos problemas que ha causado la COVID-19. El 30 de abril de 2021 cuatro estudiantes participantes expusieron los resultados del análisis de la normativa estudiada respecto de la temática de la vacunación.

Un tercer proyecto a reseñar es el desarrollado a través de un convenio de colaboración con el Ilustre Colegio de Abogados de Oviedo, con fecha 13 de febrero de 2021, que permitirá realizar actividades solidarias y de responsabilidad social relacionadas al ODS 5 (igualdad de género) y ODS 16 (paz, justicia e instituciones sólidas) y, concretamente, se investigarán las órdenes de protección para las víctimas de violencia doméstica y/o de género en un análisis estadístico sobre el número de órdenes de protección solicitadas y las acordadas en los últimos tres años, además de verificar datos como los perfiles de las víctimas, las causas de denegación, el contenido y la efectividad de las medidas acordadas, y eventuales quebrantamientos de las mismas.

Queremos destacar, también, el proyecto formalizado con la Asociación Española contra el Cáncer (AECC), con talleres destinados a personas enfermas de cáncer y sus familiares o acompañantes, impartidos por estudiantes sobre los derechos de los pacientes y de sus acompañantes, con lo cual se estudiará y analizará la normativa aplicable, además de poder dialogar con pacientes que han superado o están en fase de ello, la enfermedad, al igual que con sus familiares. De este proyecto participan trece estudiantes tanto del Grado en Derecho, como del doble Grado en Derecho y Administración de Empresas y ya se han celebrado varias reuniones conjuntas con la AECC, en las que se enseñó cómo dirigirse o tratar con personas con cáncer a través de una psicóloga y una trabajadora social de AECC, lo que ha permitido comenzar a analizar temas como el derecho a la autonomía y a la información por parte de los pacientes.

Además de la impartición de algunas charlas relacionadas con los ODS 1 (hambre cero) y con el ODS 15 (Vida de ecosistemas terrestres), cuatro proyectos han terminado: la participación en el evento Olimpiuris de Olimpiadas Jurídicas de la Universidad del Atlántico de Colombia, el Brindis solidario con el Banco de Alimentos, la colaboración con Helsinki España en el programa “Jóvenes para Jóvenes: Educando en Derechos 
Humanos y Objetivos de Desarrollo Sostenible" y la impartición de charlas de orientación académica para estudiantes de bachiller del IES Número 5 de Avilés.

Por otra parte, la Clínica del Máster en protección jurídica de las personas y de los grupos vulnerables de la Universidad de Oviedo ha colaborado en la redacción de la "Guía Jurídica Básica frente al Covid-19 (ODS 3 Salud y Bienestar)" (LÓPEZ SÁNCHEZ y CARMONA PAREDES, 2021), impulsada por la Cátedra Institucional Clínica Jurídica de la Universidad Miguel Hernández de Elche y elaborada conjuntamente por diez clínicas jurídicas, y en el informe "Libertad de Expresión, delitos de odio y VIH: a propósito de un cartel”, promovido por la Clínica de la Universidad de Alcalá en colaboración con la Coordinadora estatal para VIH y Sida (MARTÍN JIMÉNEZ y NASTAACHE, 2021).

Para el curso 2021-2022 están planificados, hasta ahora, las siguientes actuaciones: participar en el Congreso de la Red Española de Clínicas Jurídicas presentando este proyecto coordinado, colaborar en la publicación de una obra colectiva sobre "Las relaciones entre el Derecho y los Objetivos de Desarrollo Sostenible (ODS)" impulsada por la Facultad de Derecho con temática transversal para lograr una máxima colaboración con investigadora (Facultad de Derecho de la Universidad de Oviedo, 2021); realizar un taller para voluntariado con Cruz Roja; colaborar con la Fundación Padre Vinjoy, iniciar un estudio de la participación de la mujer en los órganos de representación de sociedades y un proyecto con la Fundación Secretariado Gitano sobre delitos de odio.

\subsection{Balance provisional}

Primeramente, es necesario poner de relieve que este Proyecto coordinado comenzó su andadura durante el período de la pandemia de COVID-19, lo que planteó obvias dificultades para su desenvolvimiento por la suspensión en la Universidad de Oviedo de la docencia presencial, pero esta circunstancia, lejos de ser un factor de paralización o desaliento, supuso un "antes y un después” en las actividades clínicas, en un sentido muy positivo, tanto de expansión de las relaciones con otras universidades extranjeras como un agente facilitador de la comunicación, vía correo electrónico, Teams y Zoom, entre alumnado, profesores, monitores, representantes de entidades, sociedad civil, etc. 
Igualmente, este contexto pandémico ha permitido impulsar y colaborar en actividades dirigidas a combatir algunas de sus consecuencias sociales y jurídicas.

En segundo lugar, y a pesar de los problemas mencionados, se ha conseguido la participación de un número considerable de estudiantes: 54 de los que cursan el Grado en Derecho o el Doble Grado Derecho/ADE y 5 del Máster en protección jurídica de las personas y los grupos vulnerables (en este segundo caso suponen un tercio de las personas matriculadas en el curso académico); en lo que al profesorado se refiere han colaborado 21 docentes de los tres Departamentos Jurídicos y se han sumado 16 colaboradores externos (de entidades no gubernamentales y otras universidades).

En tercer término, y por lo que respecta a la generación de conocimiento académico y social, se ha colaborado en la publicación, como ya se ha dicho, de la "Guía Jurídica Básica frente al Covid-19 y del informe "Libertad de Expresión, delitos de odio y VIH: a propósito de un cartel", siendo la primera de dichas publicaciones una muestra de notable impacto social, por el elevado número de visitas, y de trabajo en red de buena parte de las Clínicas jurídicas españolas. Están pendientes, además, varias colaboraciones que se publicarán en el citado libro colectivo que editará la Facultad de Derecho de la Universidad de Oviedo.

En cuarto lugar, cabe destacar que las actividades desarrolladas han alcanzado un alto grado de visibilidad social y académica merced al uso de diversas vías:

a) el Boletín de Noticias de la Facultad de Derecho, que se publica semanalmente y donde han aparecido las actuaciones con sus objetivos, estado y evolución, así como los distintos llamamientos que se hacen para que el estudiantado se incorpore a los proyectos;

b) la página web de la Facultad de Derecho (https://derecho.uniovi.es/organizacion/clinicajuridica/presentación), donde, además de las reglas de funcionamiento de las Clínicas, se recogen todas las noticias relacionadas con las actividades desarrolladas desde el año 2016. Este apartado se estructura en tres partes: Agenda, Informes y Presentaciones y Espacio Solidario. Este curso se han añadido 25 entradas en el apartado de agenda, 
9 en el relativo a informes y presentaciones, y 1 en el relacionado con espacio solidario;

c) las páginas de las Clínicas en Facebook y los perfiles en Twitter.

d) la Reseña de actividades en la revista Diálogos Jurídicos: Anuario de la Facultad de Derecho.

Ha de señalarse, además, la ayuda que ha brindado el Gabinete de Prensa de la Universidad de Oviedo, contribuyendo a difundir en el Diario de la Universidad de Oviedo (DUO) y en sus perfiles sociales las actividades más relevantes. En varias ocasiones los medios de comunicación social, públicos y privados, se han hecho eco de las actividades: entrevistas en la Radio-Televisión del Principado de Asturias y reportajes en los diarios La Nueva España y el Comercio (disponibles en https://derecho.uniovi.es/organizacion/clinicajuridica/medios).

En quinto término, cabe destacar las entidades sociales con las que se ha colaborado: Asociación Española contra el Cáncer, Amnistía Internacional Asturias, Fundación Secretariado Gitano, Fundación Banco de Alimentos, AFESA Salud Mental Asturias, Asociación Hierbabuena.

Finalmente, merece mencionarse, a pesar de que no se trata de un proyecto en sí, la alianza realizada con Naciones Unidas, a través de la Oficina de las Naciones Unidas contra la Droga y el Delito (UNODC), que se inserta en el ODS 17 y promueve la Educación para la Justicia-E4J, con el objeto de prevenir la delincuencia y promover una cultura de legalidad mediante actividades educativas dirigidas a la enseñanza primaria, secundaria y superior.

Pues bien, ya tenemos un resultado: el primer podcast en lengua española de los "World Podcast Series" titulado "Universidades + Agenda 2030= La juventud tomando acción", en la que el coordinador de la Clínica del Máster y una egresada del mismo y colaboradora de la Clínica cuentan sus experiencias en las Clínicas de la Universidad de Oviedo y exponen las líneas básicas de este Proyecto Conjunto de Innovación Docente: https://www.unodc.org/e4j/es/tertiary/global-podcast-series/episode-13.html 
En suma, y considerando las expectativas iniciales que parecían verse mermadas por la pandemia mundial de Covid-19, la valoración del Proyecto es muy positiva y nos anima a seguir trabajando con la metodología clínica jurídica, superando obstáculos y ayudando al estudiantado a no desconectarse de la realidad circundante, dentro de una Universidad plural, comprometida con ideales de justicia y respetuosa con sus responsabilidades sociales, con el objetivo de optimizar la transferencia de los ODS a la sociedad civil, profesional, académica, etc.

\section{Bibliografía}

AA.VV. (2011) “Clínicas Jurídicas: Desafío y oportunidad”, Revista Educación y Derecho, (4).

AA.VV. (2015) “Modelos de Clínicas Jurídicas en España”, Revista Educación y Derecho, (11).

BLOCH, F. S. (2010) The Global Clinical Movement: Educating Lawyers for Social Justice, Reino Unido: Oxford University Press.

BLOCH, F. S. (ed.) (2012) El Movimiento Global de las Clínicas Jurídicas: Formando juristas en la Justicia Social, Valencia: Tirant lo Blanch.

FACULTAD DE DERECHO DE LA UNIVERSIDAD DE OVIEDO, disponible en: Facultad de Derecho - Universidad de Oviedo - Librocolectivo - Noticias (uniovi.es), acceso el 15/'6/2021.

FERNÁNDEZ-RIVERA GONZÁLEZ, M.P. y GARCÍA ÁMEZ, J. Clínica Jurídica de la Universidad de Oviedo. Disponible en: CLÍNICA JURÍDICA DE LA FACULTAD DE DERECHO DE LA UNIVERSIDAD DE OVIEDO.pdf

FRANK, J. (1933) “Why not a clinical lawyer-school?”, University of Pennsylvania Law Review and American Law Register 81(8), pp. 907-923. 
GARCÍA AÑÓN, J. (2015) “La evolución de la Educación Jurídica Clínica en España”, Revista de Educación y Derecho. Education and Law Review (11), pp. 1-6.

LÓPEZ SÁNCHEZ, C. y CARMONA PAREDES, R. (dirs.), RAMIRO AVILÉS, M.A. (coord.) (2020), Guía Jurídica frente al Covid-19, Cátedra Institucional Clínica Jurídica de la Universidad Miguel Hernández de Elche. Disponible en: HTTPS://WWW.ACADEMIA.EDU/45026344/GU\%C3\%8DA_JUR\%C3\%8DDICA_B\%C3 \%81SICA_FRENTE_A_LA_COVID_19

MARTÍN JIMÉNEZ, B. y NASTAACHE, A. (redacción), PRESNO LINERA, M.A. y RAMIRO AVILÉS, M.A. (coordinación) y RAMÍREZ CARVAJAL, P. (revisión) (2021), Libertad de Expresión, delitos de odio y VIH: a propósito de un cartel, Universidad de Alcalá, Universidad de Oviedo, Coordinadora estatal de VIH y Sida. Disponible en: https://www.academia.edu/45650160/LIBERTAD_DE_EXPRESI\%C3\%93N_DELITO S_DE_ODIO_Y_VIH_A_PROP\%C3\%93SITO_DE_UN_CARTEL

NACIONES UNIDAS, A/RES/66/288, 27 de julio 2012, The future we want. Disponible en: $\mathrm{A} / \mathrm{RES} / 66 / 288$ : The future we want (un.org)

PRESNO LINERA, M.A. (2018) “Un proyecto de innovación docente y de transformación social: la Clínica del Máster en protección jurídica de las personas y los grupos vulnerables de la Universidad de Oviedo", Oñati Socio-Legal Series, [online] 8 (4), pp. 504-520. Disponible en: $\underline{\text { SSRN-id3126178.pdf }}$

RED ESPAÑOLA DE CLÍNICAS JURÍDICAS, disponible en QUIÉNES SOMOS (clinicas-juridicas.blogspot.com)

WITKER, J. (2007) "La enseñanza clínica como recurso de aprendizaje jurídico", Academia. Revista para la enseñanza del Derecho, 5(10), pp. 181-207. 